\title{
Polytropes and Tropical Eigenspaces: Cones of Linearity
}

\author{
Ngoc Mai Tran
}

Received: 14 May 2012 / Revised: 20 February 2013 / Accepted: 15 January 2014 /

Published online: 8 February 2014

(C) Springer Science+Business Media New York 2014

\begin{abstract}
The map which takes a square matrix $A$ to its polytrope is piecewise linear. We show that cones of linearity of this map form a polytopal fan partition of $\mathbb{R}^{n \times n}$, whose face lattice is anti-isomorphic to the lattice of complete set of connected relations. This fan refines the non-fan partition of $\mathbb{R}^{n \times n}$ corresponding to cones of linearity of the eigenvector map. Our results answer open questions in a previous work with Sturmfels (Bull. Lond. Math. Soc. 54:27-36, 2013) and lead to a new combinatorial classification of polytropes and tropical eigenspaces.
\end{abstract}

Keywords Polytrope · Tropical algebra · Eigenspace $\cdot$ Combinatorial type All-pairs shortest path

\section{Introduction}

Consider doing arithmetics in the max-plus tropical algebra $(\mathbb{R}, \oplus, \odot)$ defined by $u \oplus v=\max \{u, v\}, u \odot v=u+v$. In this case, a matrix $A \in \mathbb{R}^{n \times n}$ has a unique tropical eigenvalue $\lambda(A) \in \mathbb{R}[3,9]$. Its tropical eigenspace $\operatorname{Eig}(A)$ is the set of vectors $x$ in the tropical torus $\mathbb{T P}^{n-1}:=\mathbb{R}^{n} / \mathbb{R} \cdot(1, \ldots, 1)^{T}$ which satisfy the tropical eigenvector-eigenvalue equation

$$
\max _{1 \leq j \leq n}\left(A_{i j}+x_{j}\right)=\lambda(A)+x_{i} \quad \text { for each } i=1, \ldots, n .
$$

The set $\operatorname{Eig}(A)$ recently found applications in pairwise ranking, see [6, 14], where it appears as a special subset of solutions to the equation

$$
\max _{1 \leq i, j \leq n}\left(A_{i j}+x_{j}-x_{i}\right)=\lambda(A) .
$$


The set of $x \in \mathbb{T P}^{n-1}$ satisfying (2) is convex both in the tropical and ordinary sense. Following Joswig and Kulas [8], we term it the polytrope of $A$, denoted $\operatorname{Pol}(A)$. Both $\operatorname{Eig}(A)$ and $\operatorname{Pol}(A)$ are tropical polytopes, that is, the tropical convex hulls of finitely many points in $\mathbb{T P}^{n-1}$, as in $[5,10]$. We shall abuse terminologies and identify $\operatorname{Eig}(A)$ and $\operatorname{Pol}(A)$ with their tropical extreme points.

This paper studies conditions under which the piecewise linear maps $A \mapsto \operatorname{Eig}(A)$ and $A \mapsto \operatorname{Pol}(A)$ are given by linear functionals in the entries of $A$. In a previous joint work with Sturmfels [13], we proved the existence of $\mathcal{P}_{n}$, a partition of $\mathbb{R}^{n \times n}$ into polyhedral cones on which the map $A \mapsto \operatorname{Eig}(A)$ is linear. The open cones of $\mathcal{P}_{n}$ are in bijection with the set of connected functions. The main results of this paper, stated below, parallel and extend these theorems. The notations will be fully defined later.

Theorem 1 There exists a fan $\mathcal{F}_{n}$ such that in the relative interior of its cones, the polytrope map $A \mapsto \operatorname{Pol}(A)$ is given by a unique set of linear functionals in the entries of $A$. Furthermore, $\mathcal{F}_{n}$ is the normal fan of an $n(n-1)$-dimensional polytope in $\mathbb{R}^{n \times n}$, whose face lattice is isomorphic to the lattice of complete sets of connected relations, denoted $\mathcal{C} \mathcal{F}[n]$.

Our proof provides an explicit formula for $\mathcal{F}_{n}$, see (3). Theorem 1 addresses all the unresolved issues in [13], namely that of a combinatorial indexing for the lower dimensional cones of $\mathcal{P}_{n}$, and the existence of a natural fan refinement which preserves information on the eigenspace.

Corollary 2 The fan $\mathcal{F}_{n}$ in Theorem 1 refines the partition $\mathcal{P}_{n}$ appearing in [13]. The coarsening to $\mathcal{P}_{n}$ of the lattice isomorphism in Theorem 1 produces an orderpreserving bijection from the closed cones of $\mathcal{P}_{n}$ to the poset of compatible sets of connected relations, denoted $\mathcal{C P}[n]$.

Theorem 1 converts the study of cones in $\mathcal{F}_{n}$ and $\mathcal{P}_{n}$ to the study of $\mathcal{C F}[n]$ and $\mathcal{C P}[n]$, which essentially are collections of graphs with constraints. From these sets of graphs, one can recover the defining equations and inequalities of the corresponding cones by applying the lattice isomorphism, which is a simple computation given in Algorithm 3. Cone intersections, for example, correspond to taking the join of two elements in $\mathcal{C F}[n]$ as described in Algorithm 1. Coarser information on a cone such as its codimension is given by an explicit formula (Proposition 25).

Connections to Literature As noted in [8], it follows from [5, Proposition 18] that all polytropes arise as $\operatorname{Pol}(A)$ for some matrix $A$. This was also known to Sergeev, Schneider and Butkovic [11], and further studied in [10]. Full-dimensional polytropes, also known as tropical simplices, are of great importance to tropical polyhedral geometry, see [8] and references therein.

The face lattice of polytropes provides a natural combinatorial classification which coincides with Green's D-relation on the semigroup of square tropical matrices [7]. On the other hand, one can declare two polytropes to be equivalent if the corresponding matrices have finite entries and they lie in the relative interior of the same cone 
in $\mathcal{F}_{n}$. We term this the graphical type of a polytrope, and the former its combinatorial type. They are not directly comparable. We do not know if there exists an indexing set equivalent to $\mathcal{C} \mathcal{F}[n]$ for the combinatorial type of polytropes. Such a set may shed light on the semigroup structure of tropical matrices, a topic intimately tied in with the 'tropical' (i.e., Brazilian) origins of tropical geometry in the work of Imre Simon [12].

Organization In Sect. 2, we explicitly construct $\mathcal{F}_{n}$ and prove that it is the desired polytopal fan. In Sect. 3, we define $\mathcal{C} \mathcal{F}[n]$ and $\mathcal{C P}[n]$, the complete and compatible sets of connected relations, respectively. Section 4 proves the remaining statement of Theorem 1 and Corollary 2 by constructing the lattice anti-isomorphism $\Psi$ from $\mathcal{C F}[n]$ to the face lattice of $\mathcal{F}_{n}$. We give an algorithm to compute $\Psi$ in Sect. 5, prove a formula which gives the codimension of the cone from its graphical encoding, and discuss symmetries of the fan $\mathcal{F}_{n}$. We conclude with examples in Sect. 6 .

Notation Throughout this paper, a graph is a directed graph, allowing self-loops but not multiple edges. By a connected graph we mean weakly connected. The subgraph rooted at a node $u$ is the set of nodes and edges belonging to paths which flow into $u$. A collection of nodes $S$ in a graph $G$ is a strong component if the induced subgraph on $S$ is strongly connected. A strong component $S$ is a sink component if there are no directed edges from $S$ to its complement. We use the term multigraph to mean a directed graph with multiple distinct arcs, that is, it consists of an ordered 4-tuple ( $V, E, s, t$ ) where $V$ is the set of nodes, $E$ is the set of edges, $s: E \rightarrow V$ is a map assigning to each edge its source node, and $t: E \rightarrow V$ is a map assigning to each edge its target node. The contraction $\widetilde{G}$ of a connected graph $G$ is a multigraph whose nodes are indexed by the sink components of $G$, and whose edge set, source and target maps are induced by the edges of $G$. An in-directed tree is a tree whose edges are oriented towards the root. The graph of a matrix $A \in \mathbb{R}^{n \times n}$ is the weighted graph with edge weights $A_{i j}$. For a path $P_{i i^{\prime}}$ from $i$ to $i^{\prime}$, let $\left|P_{i i^{\prime}}\right|$ denote the number of edges, $A\left(P_{i i^{\prime}}\right)$ denote the sum of all edge weights along the path in the graph of $A$. We say that two paths are disjoint if they do not share any edges. For disjoint paths $P_{i i^{\prime}}, P_{i^{\prime} j}$, we write $P_{i i^{\prime}}+P_{i^{\prime} j}$ for their concatenation. For two fans $\mathcal{F}_{1}, \mathcal{F}_{2}$, let $\mathcal{F}_{1} \wedge \mathcal{F}_{2}$ denote their common refinement.

\section{Construction of $\mathcal{F}_{n}$}

In this section, we give an explicit construction of $\mathcal{F}_{n}$, and show that it is polytopal. We first need a small result in polyhedral geometry which gives one way of constructing a polytopal fan.

Definition 3 Let $\mathcal{F}$ be a fan refinement of a convex, full-dimensional, pointed polyhedral cone $C$ in $\mathbb{R}^{n}, v \in \mathbb{R}^{n}$ a vector in its interior. A cone of $\mathcal{F}$ is an outer cone if it is a subset of the boundary of $C$. The flattening of $\mathcal{F}$ along $v$ is the fan in $\mathbb{R}^{n-1}$ whose cones are the images of the outer cones of $\mathcal{F}$ projected onto the orthogonal complement of $v$. 
Lemma 4 Suppose $\mathcal{F}$ is the normal fan of some convex polyhedron $P$ in $\mathbb{R}^{n}$ with a pointed, full-dimensional recession cone. Let $v$ be a vector in the interior of $\mathcal{F}$. Then the flattening of $\mathcal{F}$ along $v$ is a polytopal fan in $\mathbb{R}^{n-1}$.

Proof Implicitly, the lemma claimed that $\mathcal{F}$ is a fan refinement of a full-dimensional, pointed polyhedral cone. To see this, let $P$ be the polyhedron in the lemma. Write $P=Q+C$ where $C$ is its recession cone, $Q$ a polytope. By definition, the polar $C^{\circ}$ is the normal fan of $C$. Since $C$ is full-dimensional, the facet normals of $Q$ lie in the interior of $C^{\circ}$. Thus the normal fan of $P$ is a fan refinement of the polyhedral cone $C^{\circ}$.

An $(n-1)$-dimensional unbounded face of $P$ is a face of $Q$ plus a face of $C$, hence its normal vector is an outer ray of $\mathcal{F}$. An $(n-1)$-dimensional bounded face of $P$ is necessarily a face of $Q$, whose facet normal must lie in the interior of $C^{\circ}$. Thus the outer rays of $\mathcal{F}$ are precisely the facet normals of the unbounded faces of $P$.

We now explicitly construct the polytope in question. Let $\mathcal{H}$ denote the orthogonal complement of $v$. Under the convention that the facet normals point outwards, the linear functional $x \mapsto\langle v, x\rangle$ on $\mathbb{R}^{n}$ is bounded above on $P$, and bounded below on $Q$. Thus there exists a sufficiently small $M$ such that $\langle v, x\rangle>M$ for all $x \in Q$. Let $\mathcal{H}_{M}=\mathcal{H}+M$. View $P_{M}=P \cap \mathcal{H}_{M}$ as a polytope in the $(n-1)$-dimensional affine subspace $\mathcal{H}_{M}$. We claim that $P_{M}$ is the needed polytope. Indeed, by construction, the $i$-dimensional faces of $P_{M}$ are the unbounded $(i+1)$-dimensional faces of $P$ intersected with $\mathcal{H}_{M}$. Thus it is sufficient to show that the projected outer rays of $\mathcal{F}$ along $v$ are the facet normals of the corresponding faces in $P_{M}$. Let $x$ be a vector in an $(n-2)$-dimensional face of $P_{M}, f$ the corresponding outer ray of $\mathcal{F}$, and $\Pi f$ the orthogonal projection of $f$ onto $\mathcal{H}$. Since $f-\Pi f$ is orthogonal to $\mathcal{H}$, $\langle x, f-\Pi f\rangle=0$. Thus $\langle x, \Pi f\rangle=\langle x, f\rangle=0$. Therefore, the rays $\Pi f$ are the facet normals of $P \cap \mathcal{H}_{M}$.

Let $\Gamma_{n}$ be the set of simple directed cycles on the complete digraph on $n$ nodes, including self-loops. For $C \in \Gamma_{n}$, let $\chi_{C} \in\{0,1\}^{n^{2}}$ be its incidence vector. Let $\mathcal{J}_{n}$ be the polyhedral cone of matrices in $\mathbb{R}^{n \times n}$ with no positive cycles

$$
\mathcal{J}_{n}=\left\{A \in \mathbb{R}^{n \times n}: A \cdot \chi_{C} \leq 0, C \in \Gamma_{n}\right\}
$$

Its lineality space $V_{n}$ has dimension $(n-1)$, consisting of matrices with zero-cycle sum:

$$
V_{n}=\left\{A \in \mathbb{R}^{n \times n}: A \cdot \chi_{C}=0, C \in \Gamma_{n}\right\} .
$$

For any $A \in \mathcal{J}_{n}$, define $A^{+}:=A \oplus A^{2} \oplus \cdots$, and $A^{*}:=I \oplus A^{+}$, where $I$ is the matrix with 0 on the diagonal and $-\infty$ elsewhere.

The map $A \mapsto A^{*}$ is the Kleene star map, and $A^{*}$ is the Kleene star of $A$ [10]. This map is elementwise finite if an only if $A \in \mathcal{J}_{n}$. Kleene stars provide a very neat characterization of extreme points of $\operatorname{Eig}(A)$ and $\operatorname{Pol}(A)$, as well as lending them the combinatorial interpretations crucial to our construction of $\mathcal{F}_{n}$. For a proof of the following result, see [10, Sect. 3]. 
Proposition 5 For an $n \times n$ matrix $A$, define $\bar{A}:=A \odot(-\lambda(A))$. Then the extreme tropical vertices of $\operatorname{Pol}(A)$, up to tropical scaling, are precisely the columns of $\bar{A}^{*}$, and the extreme tropical vertices of $\operatorname{Eig}(A)$ are precisely the common columns of $(\bar{A})^{+}$and $\bar{A}^{*}$.

Thus to study the polytrope map, we need to understand cones of linearity of the eigenvalue map $A \mapsto \lambda(A)$ and the Kleene star map. The former is a classic result of Cunninghame-Green [4], which we reformulate in view of Lemma 4 as follows.

Proposition 6 Let $\partial \mathcal{J}_{n}$ be the flattening of $\mathcal{J}_{n}$ along $-\mathbf{1 1}^{T}$. Define

$$
\mathcal{N}_{n}=\partial \mathcal{J}_{n}+\operatorname{span}\left\{\mathbf{1 1}^{T}\right\}+V_{n}
$$

where + denotes direct sum of subspaces. Then $\mathcal{N}_{n}$ is a polytopal fan (the normal fan of a polytope) in $\mathbb{R}^{n \times n}$. Its cones are precisely the cones of linearity of the eigenvalue map.

Proof The rays of the polar $\mathcal{J}_{n}^{\circ}$ are the incidence vectors $\left\{\chi_{C}: C \in \Gamma_{n}\right\}$. Since all faces of $\mathcal{J}_{n}$ are unbounded except the 0 vertex, we can choose any constant $M<0$ for the flattening, say, $M=-1$. Now $\mathcal{J}_{n}^{\circ} \cap \mathcal{H}_{-1}$ is the normalized cycle polytope

$$
\mathcal{C}_{n}=\operatorname{conv}\left\{\frac{1}{|C|} \chi_{C}: C \in \Gamma_{n}\right\} .
$$

By Lemma $4, \partial \mathcal{J}_{n}$ is the normal fan of $\mathcal{C}_{n}$ viewed as an $\left(n^{2}-n\right)$-dimensional polytope in the affine subspace $\mathcal{H}_{-1}$. One can check that $\mathbb{R}^{n \times n}=\mathcal{H}_{-1}+\operatorname{span}\left\{\mathbf{1 1}^{T}\right\}+V_{n}$, thus $\mathcal{N}_{n}$ is the normal fan of $\mathcal{C}_{n}$ in $\mathbb{R}^{n \times n}$. By [4], the eigenvalue function $A \mapsto \lambda(A)$ is the support function of $\mathcal{C}_{n}$ in $\mathbb{R}^{n \times n}$. Thus $\mathcal{N}_{n}$ is precisely the fan of linearity of this map.

We now consider the Kleene star map $A \mapsto A^{*}$. By definition, $A_{i j}^{*}$ is the value of the maximal path from $i$ to $j$ in the graph of $A$. For a fixed column $i^{*}$, over $\mathcal{J}_{n}$, the map $A \mapsto A_{. i^{*}}^{*}$ is precisely the solution to the classical linear program of single-target longest path (more commonly formulated as single-source shortest path) [1]:

$$
\begin{array}{ll}
\text { Maximize } & \sum_{i, j=1}^{n} A_{i j} x_{i j} \\
\text { subject to } & x_{i j} \geq 0, \quad \sum_{j=1}^{n} x_{j i}-\sum_{j=1}^{n} x_{i j}= \begin{cases}n-1 & \text { for } i=i^{*}, \\
-1 & \text { for } i \neq i^{*}\end{cases}
\end{array}
$$

This linear program is bounded if and only if $A \in \mathcal{J}_{n}$. Its constraint set is a polyhedron in $\mathbb{R}^{n \times n}$ with lineality space $V_{n}$, whose vertices are in bijection with in-directed 
spanning trees on $n$ nodes with root $i^{*}$ [1]. Thus the cones of linearity of $A \mapsto A_{\cdot i^{*}}^{*}$ form a fan partition of $\mathcal{J}_{n}$. Denote this fan $\mathcal{K}_{n, i^{*}}$. Take the common refinement over all columns $i^{*}$, we obtain the fan

$$
\mathcal{K}_{n}=\bigwedge_{i^{*}=1}^{n} \mathcal{K}_{n, i^{*}}
$$

Corollary $7 \mathcal{K}_{n}$ is the fan of linearity of the Kleene star map on $\mathcal{J}_{n}$. Furthermore, it is the normal fan of a polyhedron in $\mathbb{R}^{n \times n}$.

Proof The first statement follows from Proposition 5. For each $i^{*}, \mathcal{K}_{n, i^{*}}$ is the normal fan of a polyhedron. Thus $\mathcal{K}_{n}$ is the normal fan of the Minkowski sum of the corresponding polyhedra, see [9, Sect. 2.3].

Proof of Theorem 1, part (a) Let $\partial \mathcal{K}_{n}$ be the flattening of $\mathcal{K}_{n}$ along $-\mathbf{1 1}^{T}$. Define

$$
\mathcal{F}_{n}=\partial \mathcal{K}_{n}+\operatorname{span}\left\{\mathbf{1 1}^{T}\right\}+V_{n}
$$

By Proposition 6 and Corollary $7, \mathcal{F}_{n}$ is the fan of linearity of the polytrope map $A \mapsto \operatorname{Pol}(A)$. By Lemma 4, it is a polytopal fan in $\mathbb{R}^{n \times n}$.

The fan $\mathcal{F}_{n}$ refines $\mathcal{N}_{n}$, and inherits its lineality space $\operatorname{span}\left\{\mathbf{1 1}^{T}\right\}+V_{n}$. We often identify $\mathcal{F}_{n}$ with $\partial \mathcal{K}_{n}, \mathcal{N}_{n}$ with $\partial \mathcal{J}_{n}$, as in Example 8 and Example 27.

Example $8(n=2)$ Here $\mathcal{K}_{2}$ is the trivial fan, and $\mathcal{N}_{2}=\mathcal{P}_{2}=\mathcal{F}_{2}$. The fan $\mathcal{N}_{2}$ has three cones of codimension 0 , three cones of codimension 1 , and one cone of codimension 2. Express the matrix $A$ as the vector $(x, y, z, w)$, where $x=A_{11}$, $y=A_{22}, z=A_{12}+A_{21}, w=A_{12}-A_{21}$. Then $V_{2}=\{(x, y, z, w): x=y=z=0\}$, $\mathcal{J}_{2}=\{x, y, z \leq 0, w=0\}$ is the negative orthant, and $\partial \mathcal{J}_{2}$ is the image of the three quadrants $\{x=w=0, y, z \leq 0\},\{y=w=0, x, z \leq 0\}$ and $\{z=w=0, x, y \leq 0\}$ projected onto the subspace defined by $\{w=0, x+y+z=0\}$. This projection results in a fan partition of $\mathbb{R}^{2}$ seen in Fig. 1 .

We shall revisit this example in Sect. 6 when discussing complete sets of connected relations. In the rest of this paper, we study how the sets of graphs indexing cones in Fig. 1 can be generalized to indexing sets for cones of $\mathcal{P}_{n}$ and $\mathcal{F}_{n}$ for $n \geq 3$.

\section{Complete and Compatible Sets of Connected Relations}

For fixed $i^{*}$, the open cones of $\mathcal{K}_{n, i^{*}}$ are in bijection with in-directed spanning trees on $n$ nodes with root $i^{*}$, whose edges form the optimal paths from $j$ to $i^{*}$ for all nodes $j \in\{1, \ldots, n\}, j \neq i^{*}[1]$. One can show that the open cones of $\mathcal{K}_{n}$ are in bijection with collections of $n$ in-directed spanning trees on $n$ nodes, one rooted at each node, which satisfy following path compatibility condition. 


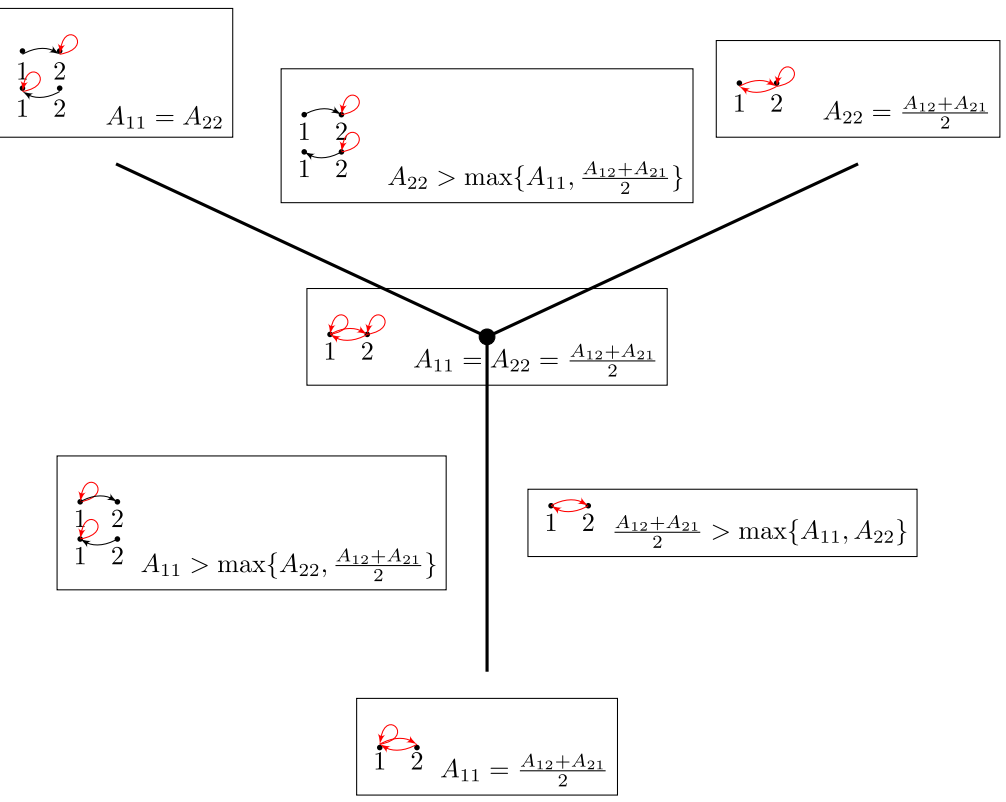

Fig. 1 Cones in the partition $\mathcal{N}_{2}=\mathcal{P}_{2}=\mathcal{F}_{2}$ of $\mathbb{R}^{2 \times 2}$, their defining equations and inequalities, and their associated maximal paths. The figure is drawn modulo lineality space, and thus represents the fan $\partial \mathcal{J}_{2}=\partial \mathcal{K}_{2}$ of $\mathbb{R}^{2}$. Edges in the critical cycles (cycles with maximal mean) are shown in red

Lemma 9 [1] If the path $i_{1} \rightarrow i_{2} \rightarrow \cdots \rightarrow i_{k}$ is a longest path from node $i_{1}$ to node $i_{k}$, then for every $q=1, \ldots, k-1$, the subpath $i_{q} \rightarrow i_{q+1} \rightarrow \cdots \rightarrow i_{k}$ is a longest path from node $i_{q}$ to node $i_{k}$.

Cones of $\mathcal{J}_{n}$ with codimension 1 are in bijection with cycles on the complete graph. Their intersections with the open cones of $\mathcal{K}_{n}$ form the open cones of $\mathcal{F}_{n}$. Thus, the later are in bijection with complete connected functions (see Definition 11). Informally, a complete connected function consists of a union of $n$ trees and a cycle, one rooted at each node, which satisfy the path compatibility condition.

Many properties of the open cones of $\mathcal{N}_{n}$ also hold for those of $\mathcal{F}_{n}$. For example, it can be shown that neighboring full-dimensional cones of $\mathcal{F}_{n}$ differ by one edge. However, due to the path compatibility condition, it is less obvious what pairs of edges can be swapped to move to a neighboring cone. Similarly, one can compute cone intersection by taking the graph union between pairs of trees with the same root node. However, in doing so, new paths (such as a cycle) may appear, which by the path compatibility condition should also be present in other trees.

Therefore, one needs an efficient indexing set for all closed cones of $\mathcal{F}_{n}$, and a join operation that corresponds to cone intersections. With the hindsight of Theorem 1, we chose to start off by defining the desired indexing set, the complete sets of connected relations. We first introduce connected relations, the building block for our indexing set. In relation to [13], they are generalizations of connected functions. 
Definition 10 A circled tree on $[n]$ is the graph union of a cycle and an in-directed spanning tree on $n$ nodes such that it has exactly one cycle. A connected relation on $[n]$ is a nonempty graph which can be written as the graph union of circled trees on $[n]$.

Let $\mathcal{R}[n]$ denote the set of all connected relations on $[n]$ adjoined with the empty set. It is a lattice ordered by subgraph inclusion, generated by its join-irreducibles, which are circled trees. Connected functions, for example, are strongly connected circled trees. Note that every connected relation has a unique strongly connected sink component. For $G \in \mathcal{R}[n]$, denote its unique sink component by $s(G)$ and the induced subgraph $S(G)$.

Definition 11 Let $\mathcal{G}=\left\{G_{1}, \ldots, G_{k}\right\}$ be a set of connected relations, $G_{i} \in \mathcal{R}[n]$. For node $u$, let $\mathcal{G}(u)$ denote the part $G_{i} \in \mathcal{G}$ containing $u$ in its sink. The list $\mathcal{G}$ is a complete set of connected relations if:

(a) The sinks $s\left(G_{j}\right), j=1, \ldots, k$ form a partition of $[n]$.

(b) For each pair $i, j \in\{1, \ldots, k\}$, let $S_{i j}$ be the subgraph of the contraction $\widetilde{G}_{j}$ of $G_{j}$ rooted at the node indexed by $s\left(G_{i}\right)$. Then $S_{i j}$ equals the induced subgraph in $\widetilde{G}_{i}$.

(c) For every triple of distinct nodes $u, v, w \in\{1, \ldots, n\}$, if there exists paths $P_{w v}, Q_{w u}$ in $\mathcal{G}(v)$ such that $Q_{w u}$ is not a subpath of $P_{w v}$, then $P_{w v}$ cannot appear in $\mathcal{G}(u)$.

Let $\mathcal{C F}[n]$ denote the collection of completed sets of connected relations.

Condition (b) is an analogue of the path compatibility condition in Lemma 9. Note that it implies that the contractions $\widetilde{G}_{i}$ have the same node set, and they are indexed by the set of sinks $s\left(G_{j}\right), j=1, \ldots k$. The need for Condition (c) is discussed after the proof of Lemma 20.

Definition 12 Write $\mathcal{G}=(\mathcal{D}, \mathcal{E})$ when we decompose $\mathcal{G}$ into the set $\mathcal{D}$ of connected relations whose sinks each contain at least one edge, and the set $\mathcal{E}$ of connected relations whose sinks each contain exactly one node and no edges. We say that $\mathcal{D}$ is a compatible set of connected relations and let $\mathcal{C P}[n]$ denote all such $\mathcal{D}$. If $\mathcal{D}$ consists of exactly one connected function, we say that $\mathcal{G}$ is a complete connected function.

Example 13 Figure 2 shows an incomplete set of connected relations $\mathcal{G}=(D, E)$ on 3 nodes. Here $s(D)=\{2\}, s(E)=\{3\}$. Since $\mathcal{G}$ is missing a part with the strong component $\{1\}$ as its sink, it is not complete.

Fig. 2 An incomplete set of connected functions $\mathcal{G}=(D, E)$

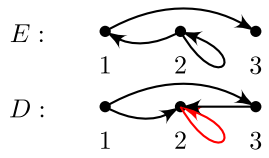



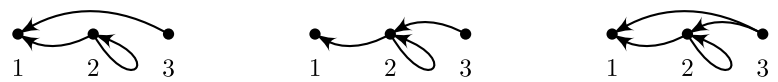

Fig. 3 All three possible connected relations that can complete the set $\mathcal{G}$ of Figure 2
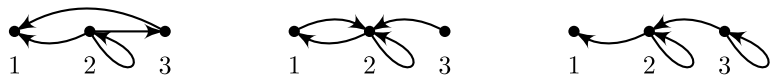

Fig. 4 Some connected relations incompatible to $\mathcal{G}$ of Figure 2

Example 14 We can complete $\mathcal{G}$ in Example 13 by adding in a connected relation with sink $\{1\}$ that does not violate Condition (b) of Definition 11. Figure 3 shows all such compatible connected relations, and Fig. 4 shows some incompatible connected relations. In the first case, the subgraph rooted at 3 consists of the edge $2 \rightarrow 3$, which is not a subgraph of $E$. The second violates Condition (a) since the sinks do not form a partition of $[n]$. The third violates Condition (b) as $D$ and $E$ do not contain the self-loop at 3.

\section{The Lattice Isomorphism}

In this section, we prove the rest of Theorem 1 , namely that the face lattice of $\mathcal{F}_{n}$ is anti-isomorphic to the lattice $\mathcal{C F}[n]$. The proof consists of three steps. First, we show that $\mathcal{C F}[n]$ is a join semilattice generated by its join irreducibles, which are the complete connected functions. Second, we produce a semilattice homomorphism $\Psi$ from $\mathcal{C F}[n]$ to a collection of cones in $\mathbb{R}^{n \times n}$ ordered by subset inclusion. Third, we show that the open cones of the fan $\mathcal{F}_{n}$ defined in (3) are precisely the images of the complete connected functions under $\Psi$. Since $\mathcal{F}_{n}$ is a fan, its face lattice ordered by subset inclusion is generated by meet-irreducibles, which are the open cones of $\mathcal{F}_{n}$. Together with the first step, this implies that $\Psi$ is a lattice anti-isomorphism between $\mathcal{C F}[n]$ and cones of $\mathcal{F}_{n}$.

\subsection{The Semilattice of $\mathcal{C F}[n]$}

We now show that $\mathcal{C F}[n]$ is a join semilattice generated by its join irreducibles, which are the complete connected functions. For $\mathcal{G} \in \mathcal{C} \mathcal{F}[n]$, let $\lambda(\mathcal{G})$ denote the partition of $[n]$ induced by the sinks of its parts. For a pair $\mathcal{G}, \mathcal{H} \in \mathcal{C} \mathcal{F}[n]$, write $\mathcal{H} \geq \mathcal{G}$ if for any pair of nodes $u$, $v$, the set of paths from $u$ to $v$ in $\mathcal{G}(v)$ is a subgraph of $\mathcal{H}(v)$. It is clear that this defines a partial ordering on $\mathcal{C F}[n]$.

We now define the join of two elements $\mathcal{G}, \mathcal{H} \in \mathcal{C F}[n]$ to be the output of Algorithm 1. This is an elaborated version of taking graph union and updating the strongly connected sink components at the same time. Broadly speaking, the algorithm iterates over three steps. First, we take the minimal amount of graph union required to satisfy Condition (a) of Definition 11. In doing so, new cycles may form which may connect two sink components, requiring them to be merged. Thus in step two, we check for all such cycles. In step three, we find all triples violating Condition (c) of Definition 11 , and resolve them by taking the graph union of the corresponding connected 


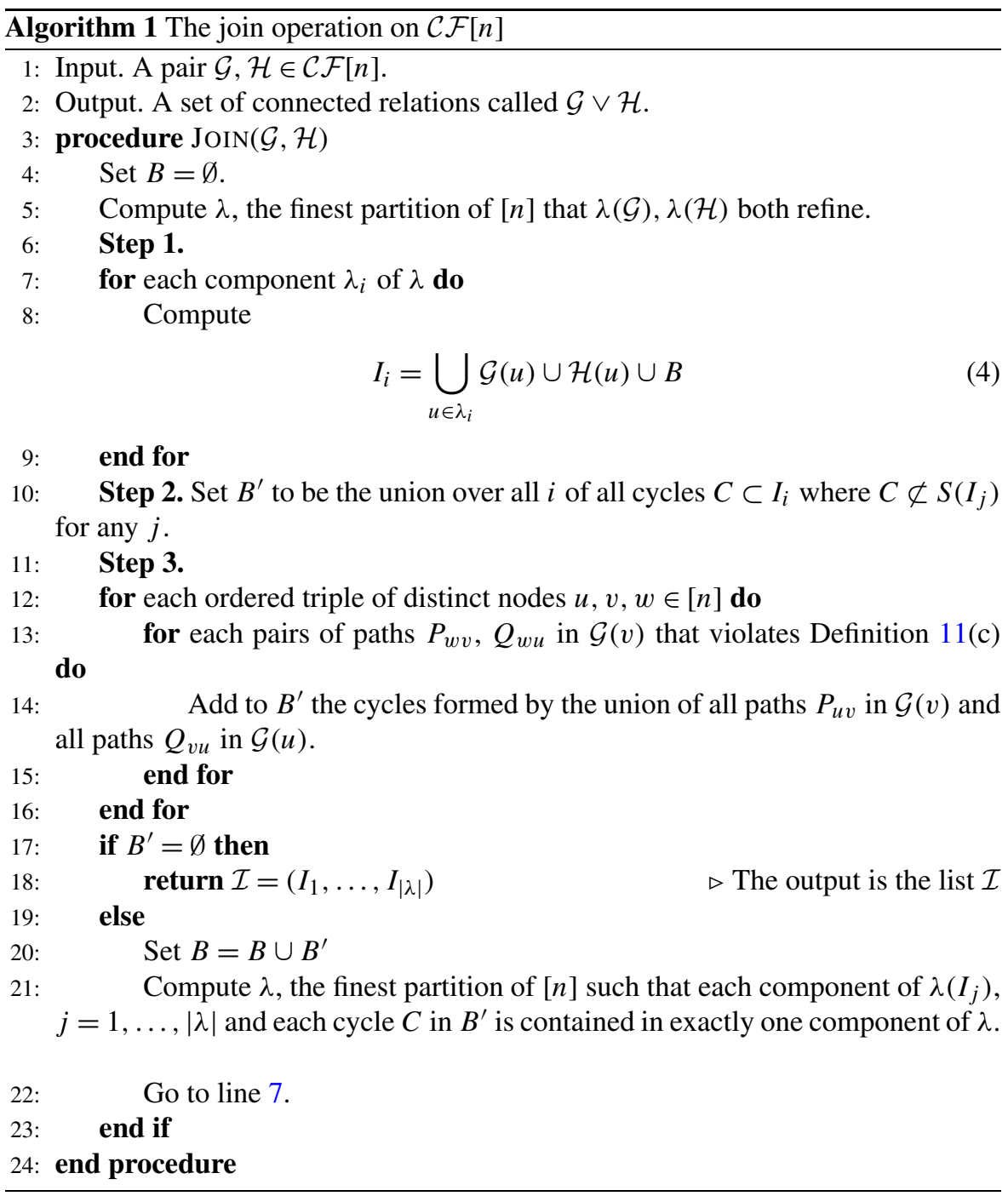

relations. If 'actions' happened in steps 2 and 3, we go back to step one. Otherwise, the algorithm terminates and outputs its current set of connected relations.

Note that after each iteration of line 21 the partition $\lambda$ is unique and becomes coarser. Thus the algorithm is well-defined and terminates. By construction, the output satisfies Definition 11, and hence it is a complete set of connected relations.

Lemma $15 \mathcal{G} \vee \mathcal{H}$ is the join of $\mathcal{G}$ and $\mathcal{H}$ in $\mathcal{C} \mathcal{F}[n]$.

Proof From construction it is clear that $\mathcal{G} \vee \mathcal{H} \in \mathcal{C F}[n]$ and $\mathcal{I} \geq \mathcal{G}, \mathcal{H}$. To see that it is the smallest such element in $\mathcal{C F}[n]$, let $\mathcal{I}^{(1)}, \mathcal{I}^{(2)}, \ldots, \mathcal{I}^{(k)}=\mathcal{I}$ be the sequence of 
intermediate candidate sets produced after the end of line 9 for each time this line is reached.

Let $\mathcal{I}^{\prime} \in \mathcal{C F}[n]$ be such that $\mathcal{I}^{\prime} \geq \mathcal{G}, \mathcal{H}$. Then each component of $\lambda(\mathcal{G}), \lambda(\mathcal{H})$ is necessarily contained in exactly one component of $\lambda\left(\mathcal{I}^{\prime}\right)$. Thus any path in $\mathcal{I}^{(1)}(v)$ is a subgraph of $\mathcal{I}^{\prime}(v)$, and in particular, any cycle $C$ in $B^{\prime}$ must be a subgraph of some part $\mathcal{I}_{i}^{\prime}$. Since $C$ is a cycle, by Definition 11 , it is contained in exactly one component of $\lambda(\mathcal{J})$. Thus any path in $\mathcal{I}^{(2)}(v)$ must be a subgraph of $\mathcal{I}^{\prime}(v)$. Iterating this argument up the sequence $\mathcal{I}^{(j)}, j=1, \ldots, k$, we see that any path in $\mathcal{I}(v)$ must be a subgraph of $\mathcal{I}^{\prime}(v)$, so $\mathcal{I}^{\prime} \geq \mathcal{I}$.

By the same argument, one can check that the operation $\vee$ is idempotent, commutative and associative. We make use of associativity in the proof of the following proposition.

Proposition $16 \mathcal{C F}[n]$ is a join semilattice generated by complete connected functions, which are precisely its sets of join-irreducibles.

Proof The join operation of Lemma 15 turns $\mathcal{C F}[n]$ into a join semilattice. It is clear that every complete connected function is join-irreducible. Thus it is sufficient to show that they generate $\mathcal{C F}[n]$.

For $\mathcal{G} \in \mathcal{C F}[n]$, let $L$ be the collection of complete connected function $\mathcal{H}$ such that $\mathcal{H} \leq \mathcal{G}$, and define $\mathcal{I}:=\bigvee_{\mathcal{H} \in L} \mathcal{H}$. By definition $\mathcal{I} \leq \mathcal{G}$. To show that $\mathcal{G} \leq \mathcal{I}$, for any pair of nodes $u, v \in[n]$, fix a path $P_{u v}$ in $\mathcal{G}(v)$. We shall construct a complete connected function $\mathcal{H}$ such that $\mathcal{H}(r) \subseteq \mathcal{G}(r)$ for any node $r \in[n]$, and $P_{u v} \subseteq \mathcal{H}(v)$. This implies $\mathcal{H} \in L, P_{u v} \subseteq \mathcal{I}(v)$, and hence $\mathcal{G} \leq \mathcal{I}$ by definition of the partial order on $\mathcal{C} \mathcal{F}[n]$. We consider two cases.

Case $1,|\lambda(\mathcal{G})|=1$. That is, $\mathcal{G}$ consists of only one part $G$ whose graph is strongly connected. It is sufficient to construct the contraction $\mathcal{H}(v)$, thus we can assume without loss of generality that $G$ contains a self-loop at $v$. Order the nodes so that $v=1$. Let $\mathcal{H}$ be the output of Algorithm 2 .

By construction the parts of $\mathcal{H}$ are in-directed spanning trees on distinct root nodes, each containing exactly one cycle which is the self-loop at node $v=1$. Thus Conditions (a) and (c) of Definition 11 are satisfied. Condition (b) follows from line 5. Thus $\mathcal{H}$ is a complete connected function, and $\mathcal{H} \leq \mathcal{G}, P_{u v} \subseteq \mathcal{H}(v)$ by construction.

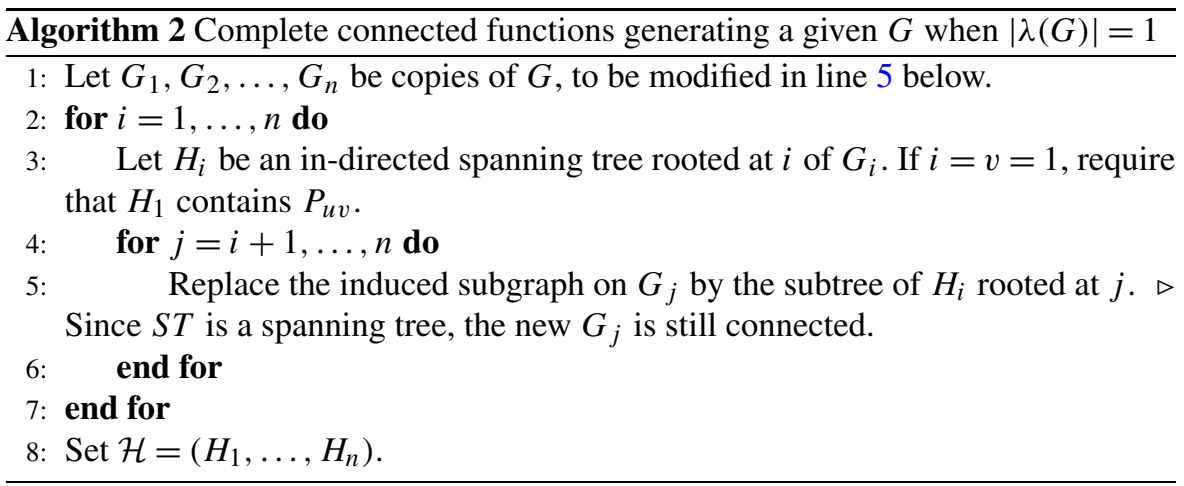


Case 2, $|\lambda(\mathcal{G})|>1$. Again, we can assume, without loss of generality, that there exists a self-loop at $v$. We first apply the above algorithm to the contractions $\widetilde{G}_{1}, \ldots, \widetilde{G}_{k}$, producing $k$ compatible in-directed spanning trees $T_{1}, \ldots, T_{k}$ on the strong components of the $G_{i}$ 's. Each edge in these trees fix a source and target node between two strong components. Now we apply the above algorithm to each strong component to split them into compatible spanning trees on singleton sinks. Finally, for each $T_{i}$, we replace each of its node (which currently is a strong component) by the corresponding spanning tree of that strong component, whose sink is specified by the edges of $T_{i}$. By construction, the collection of resulting $n$ trees on the $n$ singleton sinks is the desired complete connected function.

\subsection{From Connected Relations to Equations and Inequalities}

We now define the map which takes an element of $\mathcal{R}[n]$ to a cone in $\mathbb{R}^{n \times n}$. We then extend its domain to complete sets of connected relations, resulting in a candidate for the lattice anti-isomorphism of Theorem 1.

Definition 17 Let $\psi: G \mapsto \psi(G)$ be the map from $\mathcal{R}[n]$ to cones in $\mathbb{R}^{n \times n}$, where $\psi(G)$ is the cone defined by the linear equations and inequalities output by Algorithm 3 .

Lemma 18 Algorithm 3 is well-defined: its output is independent of the choice of $C$ and the spanning tree $T$.

Proof For any cycle $C^{\prime} \in G$, the algorithm outputs $\bar{A}\left(C^{\prime}\right)=0$, and hence $A(C) /|C|=$ $A\left(C^{\prime}\right) /\left|C^{\prime}\right|$ for all pairs of cycles $C, C^{\prime} \in G$. Thus the algorithm output is independent of the choice of the cycle $C$.

To show that the algorithm output is independent of $T$, it is sufficient to prove that for any path $P_{u r}$ from $u$ to $r$, we have $\bar{A}\left(P_{u r}\right)=\bar{A}\left(T_{u}\right)$. Indeed, suppose that this

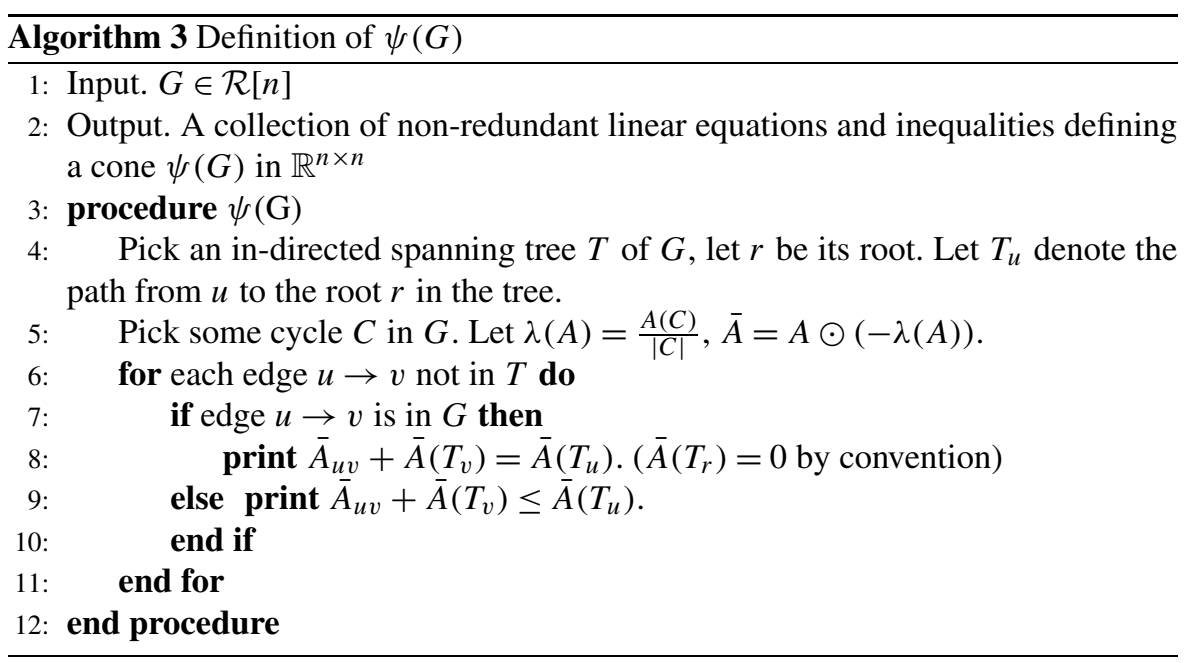


is true. Let $T^{\prime}$ be another in-directed spanning tree of $G$ with root $r^{\prime}$. Then $r, r^{\prime}$ are strongly connected, hence $\bar{A}\left(T_{u}\right)=\bar{A}\left(T_{u}^{\prime}\right)+\bar{A}\left(T_{r^{\prime}}\right)$. Thus the linear inequalities and equalities outputted are the same, since the term $\bar{A}\left(T_{r^{\prime}}\right)$ cancels on both sides.

We now prove the above claim. Fix a path $P_{u r}$, and without loss of generality, suppose that $P_{u r} \neq T_{u}$, and that the first edge of the two paths differ, that is, the edge $u \rightarrow v$ on the path $P_{u r}$ is not in $T$. Thus

$$
\bar{A}\left(T_{u}\right)=\bar{A}_{u v}+\bar{A}\left(T_{v}\right)=\bar{A}\left(P_{u r}(u \rightarrow v)\right)+\bar{A}\left(T_{v}\right) .
$$

Therefore, it is sufficient to prove that $\bar{A}\left(T_{v}\right)=\bar{A}\left(P_{u r}(v \rightarrow r)\right)$ for the induced path from $v$ to $r$ in $P_{u r}$. Repeating this argument, noting that at each step the length of the remaining path is decreasing, we see that $\bar{A}\left(P_{u r}\right)=\bar{A}\left(T_{u}\right)$.

Lemma 19 Algorithm 3 outputs the minimal set of equations and inequalities defining the cone $\psi(G)$. This is a closed, non-trivial cone, and its codimension is between 0 and $n(n-1)$. The cone $\psi(G)$ has codimension 0 if and only if $G$ is a connected function, in which case it is the cone $\Omega_{G}$ of $\mathcal{P}_{n}$ as defined in [13].

Proof The output of Algorithm 3 is minimal since the $n(n-1)$ equations and inequalities in the output are linearly independent. Indeed, each edge $u \rightarrow v$ in $G$ gives rise to the unique equation or inequality in the output that contains the entry $A_{u v}$.

The $n$-dimensional lineality space of $\mathcal{N}_{n}$ is contained in $\psi(G)$ for any $G \in \mathcal{R}[n]$. Thus $\psi(G)$ is a closed, non-trivial cone, with codimension between 0 and $n(n-1)$. Algorithm 3 outputs no equality if and only if $G$ is a circled tree with tree $T$ and cycle $C$. In this case, the output of Algorithm 3 is precisely the $n(n-1)$ facet-defining inequalities of the cone $\Omega_{G}$ found in [13].

Unfortunately, $\psi$ does not completely carry over the lattice structure of $\mathcal{R}[n]$ to its image set of cones.

Lemma 20 Suppose $G, H \in \mathcal{R}[n]$. Then $\psi(G \cup H) \subseteq \psi(G) \cap \psi(H)$. Furthermore, $\psi(G \cup H)=\psi(G) \cap \psi(H)$ if and only if either:

(a) The sink components are not disjoint, or

(b) There exists a triple of distinct nodes $u, v, w, u \in s(G), v \in s(H)$ and paths $P_{w v}, Q_{w u}$ in both $G$ and $H$ such that $Q_{w u} \neq P_{w v}(w \rightarrow u)$.

Proof The first statement follows from the definition of $\psi$. We prove the second statement by considering three disjoint cases: when Condition (a) holds, when (a) does not hold but (b) holds, and when neither (a) nor (b) holds.

Suppose that (a) holds, that is, $s(G) \cap s(H) \neq \emptyset$. Any inequality in $\psi(G \cup H)$ is also an inequality in both $\psi(G)$ and $\psi(H)$. Thus for $A \in \psi(G) \cap \psi(H)$, we need to show that $A$ satisfies the extra equations in $\psi(G \cup H)$. Let $r \in s(G) \cap s(H)$. For $u \in[n]$, consider the two paths from $u$ to $r: G_{u r}$ and $H_{u r}$, coming from $G$ and $H$ respectively. Since $A \in \psi(G), \bar{A}\left(G_{u r}\right) \geq \bar{A}\left(H_{u r}\right)$. Since $A \in \psi(H)$, $\bar{A}\left(G_{u r}\right) \leq \bar{A}\left(H_{u r}\right)$. Thus $\bar{A}\left(G_{u r}\right)=\bar{A}\left(H_{u r}\right)$ for all $u \in[n]$, so $A \in \psi(G \cup H)$. 
Suppose $s(G) \cap s(H)=\emptyset$ and Condition (b) holds. By induction on the number of nodes, we reduce to the case $n=3$ with $s(G)=\{1\}, s(H)=\{2\}$, and the triple $1,2,3$ witnesses Condition (b). That is, $G$ contains the edges $2 \rightarrow 1,3 \rightarrow 1,3 \rightarrow 2$, and $H$ contains the edges $1 \rightarrow 2,3 \rightarrow 1,3 \rightarrow 2$. Let $A \in \psi(G) \cap \psi(H)$. By definition of $\psi, A_{11}=A_{22}=\lambda(A)$. Since $A \in \psi(G), \bar{A}_{31}=\bar{A}_{32}+\bar{A}_{21}$. But $A \in \psi(H)$, hence $\bar{A}_{32}=\bar{A}_{31}+\bar{A}_{21}$. Thus $\bar{A}_{21}+\bar{A}_{12}=0$, that is, the cycle $C=1 \rightarrow 2 \rightarrow 1$ is also a critical cycle of $A$. Thus

$$
\psi(G) \cap \psi(H)=\psi(G \cup C) \cap \psi(H \cup C)=\psi((G \cup C) \cup(H \cup C))=\psi(G \cup H),
$$

where the last equality follows from the first case.

Suppose neither Condition (a) nor Condition (b) holds. By induction on the number of nodes, we can reduce to the case $n=2$, where $s(G)=\{1\}, s(H)=\{2\}$. By enumerating all possibilities, we can check that regardless of the critical cycle, the matrix

$$
A=\left[\begin{array}{cc}
0 & -1 \\
-1 & 0
\end{array}\right]
$$

lies in $\psi(G) \cap \psi(H)$, but not $\psi(G \cup H)$, for example.

We now show that one can extend $\psi$ to the map $\Psi$ from $\mathcal{C F}[n]$ to cones in $\mathbb{R}^{n \times n}$ satisfying $\Psi(\mathcal{G} \vee \mathcal{H})=\Psi(\mathcal{G}) \cap \Psi(\mathcal{H})$. This will be our lattice anti-isomorphism between $\mathcal{C} \mathcal{F}[n]$ and the fan $\mathcal{F}_{n}$.

Definition 21 Let $\mathcal{G}=\left(G_{1}, \ldots, G_{k}\right)$ where each $G_{i} \in \mathcal{R}[n]$. Define a cone in $\mathbb{R}^{n \times n}$ by

$$
\Psi(\mathcal{G})=\bigcap_{i=1}^{k} \psi\left(G_{i}\right) .
$$

Proposition 22 For $\mathcal{G}, \mathcal{H} \in \mathcal{C} \mathcal{F}[n]$,

$$
\Psi(\mathcal{G} \vee \mathcal{H})=\Psi(\mathcal{G}) \cap \Psi(\mathcal{H})
$$

Proof The ' $\subseteq$ ' direction follows from Lemma 20. For the other direction, consider the sequence $\left(B_{1}^{\prime}, B_{2}^{\prime}, \ldots, B_{k}^{\prime}=\emptyset\right)$ at line 17 of Algorithm 1 . Let $\left(\lambda_{1}, \ldots, \lambda_{k}\right)$ be the corresponding sequence of partition used at the start of line 7 . We shall prove the ' $\supseteq$ ' direction by induction on $k$.

If $k=1, B_{1}^{\prime}=\emptyset$. Since the parts of $\mathcal{G}$ and $\mathcal{H}$ are connected, this cannot happen when the union of line 7 was taken over components with disjoint sinks. Thus $\lambda(\mathcal{G})=$ $\lambda(\mathcal{H})=\lambda(\mathcal{G} \vee \mathcal{H})=\lambda$. By Lemma 20,

$$
\begin{aligned}
\Psi(\mathcal{G} \vee \mathcal{H}) & =\bigcap_{i=1}^{|\lambda|} \psi\left(\bigcup_{u \in \lambda_{i}} \mathcal{G}(u) \cup \mathcal{H}(u)\right)=\bigcap_{i=1}^{|\lambda|} \bigcap_{u \in \lambda_{i}} \psi(\mathcal{G}(u)) \cap \psi(\mathcal{H}(u)) \\
& =\Psi(\mathcal{G}) \cap \Psi(\mathcal{H}) .
\end{aligned}
$$


If $k=2$, each cycle $C \in B_{1}^{\prime}$ was generated by union of parts in $\mathcal{G}$ and $\mathcal{H}$ that either have non-disjoint sinks, or have the triple-node situation. These are precisely the two cases covered by Lemma 20. Thus for any $A \in \Psi(\mathcal{G}) \cap \Psi(\mathcal{H}), \bar{A}(C)=0$, and the cone defined by $\Psi(\mathcal{G}) \cap \Psi(\mathcal{H})$ is unchanged when we adjoin each cycle $C \in B_{1}^{\prime}$ to each of the parts of $\mathcal{G}$ and $\mathcal{H}$. Call this new set $\mathcal{G}^{\prime}$ and $\mathcal{H}^{\prime}$, that is, define $\mathcal{G}^{\prime}(u)=\mathcal{G}(u) \bigcup_{C \in B_{1}^{\prime}} C, \mathcal{H}^{\prime}(u)=\mathcal{H}(u) \bigcup_{C \in B_{1}^{\prime}} C$. Now suppose that there are nodes $u, v \in C$ which lie in different components of $\lambda_{1}$. But $u, v \in s\left(\mathcal{G}^{\prime}(u)\right) \cap s\left(\mathcal{H}^{\prime}(u)\right)$, thus

$$
\Psi(\mathcal{G}(u)) \cap \Psi(\mathcal{H}(u))=\Psi\left(\mathcal{G}^{\prime}(u)\right) \cap \Psi\left(\mathcal{H}^{\prime}(u)\right)=\Psi\left(\mathcal{G}^{\prime}(u) \cup \mathcal{H}^{\prime}(u)\right)
$$

Therefore, $\Psi\left(\mathcal{G}^{\prime}\right) \cap \Psi\left(\mathcal{H}^{\prime}\right)=\Psi(\mathcal{G}) \cap \Psi(\mathcal{H})$. Since $B_{2}^{\prime}=\emptyset$, it follows that

$$
\Psi(\mathcal{G} \vee \mathcal{H})=\Psi\left(\mathcal{G}^{\prime}\right) \cap \Psi\left(\mathcal{H}^{\prime}\right)
$$

Combining the last two equations proves the case $k=2$. Since the algorithm terminates in at most $n^{2}$ steps, $k \leq n^{2}$, and induction completes the proof.

Proposition $23 \Psi$ defines a bijection from the join-irreducibles of $\mathcal{C F}[n]$ to the closed, full-dimensional cones of $\mathcal{F}_{n}$.

Proof By construction of $\mathcal{F}_{n}$, its closed, full-dimensional cone are in bijection with a collection of $n$ trees of longest paths on the $n$ roots, each union with a cycle $C$. Note that trees with roots in $C$ coincide. Thus the set of such trees are precisely the complete connected function, which are the join-irreducibles of $\mathcal{C F}[n]$. Finally, $\Psi$ maps a complete connected function to the corresponding cone in $\mathcal{F}_{n}$ by definition of $\psi$.

Proof of Theorem 1 (lattice anti-isomorphism statement) Propositions 16 and 23 show that $\Psi$ is a bijection from the join-irreducibles of the finitely generated semilattice $\mathcal{C} \mathcal{F}[n]$ to the meet-irreducibles of the face lattice of $\mathcal{F}$. It follows from Proposition 22 that $\Psi$ is a lattice anti-isomorphism. This concludes the proof of Theorem 1 .

Proof of Corollary 2 Let $\mathcal{G}=\left(G_{1}, \ldots, G_{k}\right)$ be a complete set of connected relations. Let $A \in \Psi(\mathcal{G})$. By Proposition 5, the sinks $s\left(G_{i}\right), i=1, \ldots, k$ index distinct tropical vertices of $\operatorname{Pol}(A)$. Write $\mathcal{G}=(\mathcal{D}, \mathcal{E})$, where $\mathcal{D}$ is the collection of complete set of connected relations whose sink contain a critical cycle. Thus the corresponding columns of $\operatorname{Pol}(A)$ are precisely the extreme tropical eigenvectors of $\operatorname{Eig}(A)[2,3]$. Therefore, compatible set of connected relations index cones of linearity of the tropical eigenvector map, which are closed cones of $\mathcal{P}_{n}$.

\section{Codimension, Facet-Defining Equations and Symmetries}

It follows from the proof of Theorem 1 that one can obtain the defining equations and inequalities of cones in $\mathcal{F}_{n}$ from their complete connected relations $\mathcal{G}$ by computing 


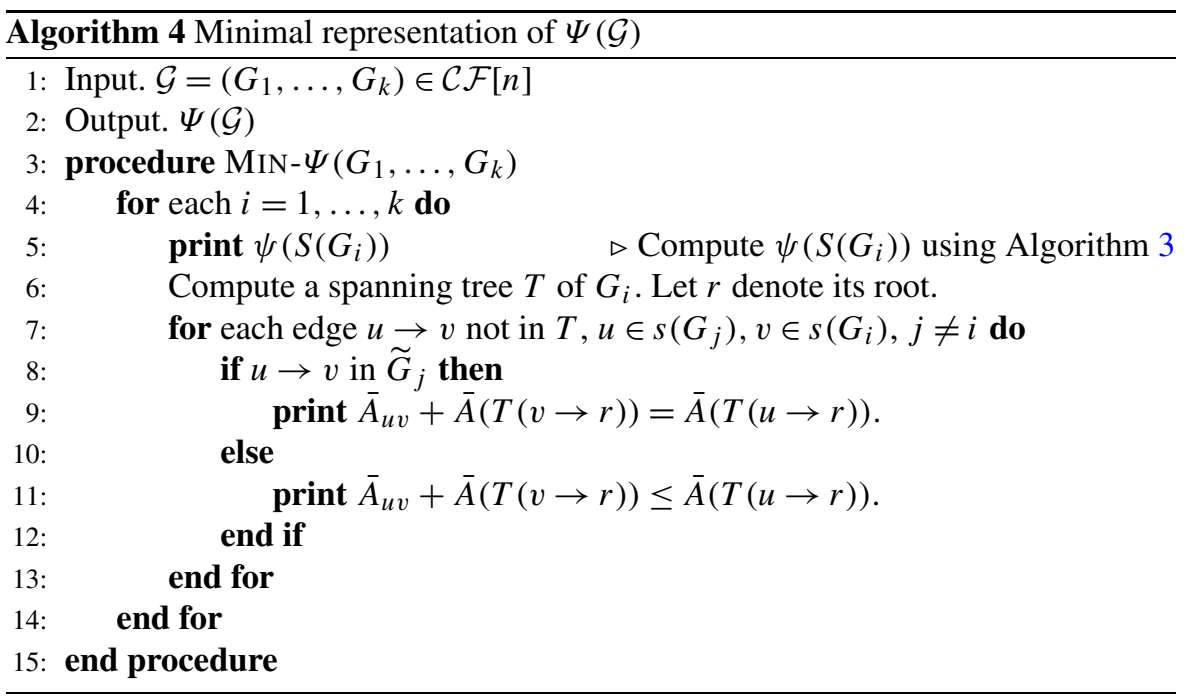

$\Psi(\mathcal{G})$. One method is to apply Algorithm 3 to obtain $\psi\left(G_{i}\right)$ for each part $G_{i}$ of $\mathcal{G}$, then compute their intersections. Here we present a more self-contained algorithm and show how one can quickly compute the codimension of $\Psi(\mathcal{G})$. The proof of minimal representation similar to that of Algorithm 3 and hence omitted.

Lemma 24 Algorithm 4 outputs a minimal set of equations and inequalities defining the cone $\Psi(G)$, and is independent of the choice of the spanning tree $T$.

Proposition 25 Let $\mathcal{G}=\left(G_{1}, \ldots, G_{k}\right) \in \mathcal{C F}[n]$. The codimension of the cone $\Psi(\mathcal{G})$ is

$$
\operatorname{codim}(\Psi(\mathcal{G}))=\sum_{i=1}^{k}\left(e_{i}-v_{i}+E_{i}-V_{i}\right),
$$

where $v_{i}, e_{i}$ are the number of nodes and edges of the graph $S\left(G_{i}\right), V_{i}$ is the number of nodes in the contraction $\widetilde{G}_{i}$ with an outgoing edge to the node indexed by $s\left(G_{i}\right)$, and $E_{i}$ is the total out-degree of such nodes. In particular, the maximal codimension of $\mathcal{G}$ is $n(n-1)$, and this happens when $G$ is the complete graph on $n$ nodes with $n$ self-loops.

Proof The codimension of $\Psi(\mathcal{G})$ is the number of equalities returned by Algorithm 4 . As in the proof of Proposition 16, we consider two cases.

Case $1, k=1$. Here $E=V=1$ and $\Psi(\mathcal{G})=\psi(G)$. The spanning tree $T$ of $G$ consists of $v-1$ edges, each other edge in $G$ contributes one equality in the output of Algorithm 3, except for the special edge $r \rightarrow v^{*}$. Thus $\operatorname{codim}(\Psi(\mathcal{G}))=e-(v-$ 1) $-1=e-v$. 
Case 2, $k>1$. By Case 1, the number of equalities produced from computing $\psi\left(S\left(G_{i}\right)\right)$ is $e_{i}-v_{i}$. Thus it is sufficient to show that lines 6 to 13 of Algorithm 4 yield $\sum_{i=1}^{k}\left(E_{i}-V_{i}\right)$ equalities. Suppose $s\left(G_{j}\right)$ has an outgoing edge to $s\left(G_{i}\right)$ in $\widetilde{G}_{i}$. If this is its unique outgoing edge, then it must be in the spanning tree $T$, yielding no equality. Otherwise, since $\widetilde{G}_{i}$ is weakly connected, each outgoing edge from $s\left(G_{j}\right)$ yields a unique alternative path from $s\left(G_{j}\right)$ to $s\left(G_{i}\right)$. Hence the number of equalities each such $s\left(G_{j}\right)$ contributes is exactly its number of outgoing edges minus 1 . Taking the sum over all such $j$, we obtain $\sum_{i=1}^{k}\left(E_{i}-V_{i}\right)$.

\subsection{Symmetries of the Fan $\mathcal{F}_{n}$}

The automorphism group of the complete directed graph on $n$ vertices consists of vertex permutations and reversing edge orientations. Vertex permutations induce symmetries on the fan $\mathcal{F}_{n}$, mapping one open cone to another. For edge reversals, note that $\operatorname{Pol}(A)=\operatorname{Pol}\left(A^{T}\right)$ up to vertex permutations $[5,8]$, thus edge reversals coincide with some vertex permutations. In terms of $\mathcal{C} \mathcal{F}[n]$, edge reversals correspond to tracking all-pairs longest paths by the sources rather than the sinks. If we take $\mathcal{G} \in \mathcal{C F}[n]$, reverse all arrows, and then group the paths by their sink, we obtain another element $\mathcal{H} \in \mathcal{C F}[n]$, and $\mathcal{G}=\mathcal{H}$ up to vertex permutations. This is a non-obvious symmetry of the fan $\mathcal{F}_{n}$. See Example 27.

\section{Examples}

Example 26 ( $n=2$, continued) In this case, $\mathcal{N}_{2}=\mathcal{P}_{2}=\mathcal{F}_{2}$, and the cones indexed by complete sets of connected functions are shown in Fig. 1. We shall refer to the cones by their critical cycles. Along the cone $C_{11,22}$ defined by $A_{11}=A_{22}>\left(A_{12}+\right.$ $\left.A_{21}\right) / 2, \operatorname{dim}(\operatorname{Eig}(A))=1$, and the two extreme tropical eigenvectors has types shown in the upper-left box of Fig. 1. Even in this small example we can see Lemma 20 in action: matrices in the relative interior of the cone $C_{11,22}$ are precisely the matrices in $\psi\left(C_{11}\right) \cap \psi\left(C_{22}\right)$ but not $\psi\left(C_{11} \cup C_{22}\right)$, and this is precisely due to the lack of uniqueness of the tropical eigenvector for matrices in this region.

Example $27(n=3)$ The $f$-vector of $\mathcal{F}_{3}$ is $(68,207,267,186,72,14,1)$. As a sanity check, note that $\mathcal{F}_{3}$ has a lineality space of dimension 3 . Identifying it with a complete pointed fan in $\mathbb{R}^{6}$, we see that the Euler characteristic of the $f$-vector of $\mathcal{F}_{3}$ excluding the point of codimension 6 should be that of a 5 -sphere, and it is indeed 0 .

Figure 5 shows $N(k, \lambda, p, c)$, the number of cones in $\mathcal{F}_{3}$ of a given dimension $k$ whose indexing complete set of connected relations $\mathcal{G}$ satisfy $\lambda(\mathcal{G})=\lambda$, with $p$ number of equalities coming from paths, and $c$ number of equalities coming from cycles. For example, $N(2,(1,1,1), 1,1)=90$ is the number of cones in $\mathcal{F}_{3}$ which has critical cycles of length at most 1 , codimension 2 and thus two defining equations, out of which one comes from a pair of distinct paths to a critical vertex, and one comes from the existence of two critical cycles. For $\lambda(\mathcal{G})=(3)$, that is, $\mathcal{G}$ has a 3 -cycle, we omit $p$ and $c$ since any equality can be regarded as a cycle equality. 


\begin{tabular}{|c|c|c|c|c|c|c|c|c|c|c|c|c|}
\hline \multicolumn{2}{|c|}{$\mathrm{k}=0$} & \multicolumn{2}{|c|}{1} & \multicolumn{2}{|c|}{2} & \multicolumn{2}{|c|}{3} & \multicolumn{2}{|c|}{4} & \multicolumn{2}{|c|}{5} & 6 \\
\hline$\lambda=(1,1,1) \quad(p, c)=0,0$ & 54 & $\begin{array}{l}1,0 \\
0,1\end{array}$ & $\begin{array}{l}90 \\
54\end{array}$ & $\begin{array}{l}2,0 \\
1,1 \\
0,2\end{array}$ & $\begin{array}{l}45 \\
90 \\
18\end{array}$ & $\begin{array}{l}3,0 \\
2,1 \\
1,2\end{array}$ & $\begin{array}{c}6 \\
45 \\
30\end{array}$ & $\begin{array}{l}3,1 \\
2,2\end{array}$ & $\begin{array}{c}6 \\
15\end{array}$ & 3,2 & 2 & \\
\hline$(2,1)$ & 12 & $\begin{array}{l}1,0 \\
0,1\end{array}$ & $\begin{array}{l}12 \\
36\end{array}$ & $\begin{array}{l}2,0 \\
1,1 \\
0,2\end{array}$ & $\begin{array}{c}3 \\
36 \\
36 \\
\end{array}$ & $\begin{array}{l}2,1 \\
1,2 \\
0,3\end{array}$ & $\begin{array}{c}9 \\
36 \\
12 \\
\end{array}$ & $\begin{array}{l}2,2 \\
1,3\end{array}$ & $\begin{array}{c}9 \\
12\end{array}$ & 2,3 & 3 & \\
\hline (3) & 2 & & 15 & & 39 & & 48 & & 30 & & 9 & 1 \\
\hline Total & 68 & & & & & & & & & & & 1 \\
\hline
\end{tabular}

Fig. 5 Table of $N(k, \lambda, p, c)$, the number of cones in $\mathcal{F}_{3}$ of a given dimension $k$ whose indexing complete set of connected relations $G$ satisfy $\lambda(G)=\lambda$, with $p$ the number of equalities coming from paths, and $c$ the number of equalities coming from cycles

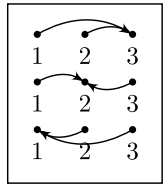

$\mathcal{G}_{1}, 1$

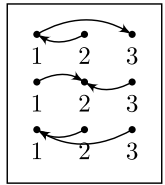

$\mathcal{G}_{2}, 6$

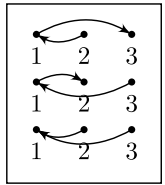

$\mathcal{G}_{3}, 3$

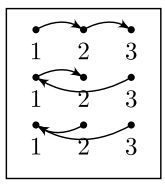

$\mathcal{G}_{4}, 6$

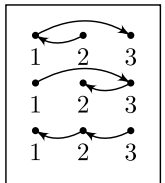

$\mathcal{G}_{5}, 2$

Fig. 6 The five equivalence classes and their sizes, $\lambda=(1,1,1), p=c=0$

The 54 cones with partition $\lambda=(1,1,1), p=0, c=0$ refine the three cones of $\mathcal{N}_{3}$ corresponding to the self-loops at 1,2 and 3. Ignoring the self-loops, we have 18 cones, with 5 equivalence classes up to permutations (and edge reversals). These equivalence classes and their sizes are shown in Fig. 6.

Suppose we reverse all edges of $\mathcal{G}_{3}$. Then we obtain three out-directed trees. Reorganizing the paths in these trees by their sinks, we obtain back $\mathcal{G}_{3}$. Thus edge reversal acts trivially on the orbit of $\mathcal{G}_{3}$. For $\mathcal{G}_{4}$, edge reversal coincides with the permutation (12).

Example 28 (Face lattice of a full-dimensional cone) We enumerate the face lattice of a full-dimensional cone in $\mathcal{F}_{3}$ indexed by the complete connected function $\mathcal{G}$ shown in the top solid box of Fig. 7. The $f$-vector of this cone is $1,6,15,20,15,6,1$. It is a cone over a 5-dimensional simplex with a 3-dimensional lineality space. Algorithm 4 gives the following set of defining equations and inequalities for $\Psi(\mathcal{G})$. For clarity we express them as formulas in $\bar{A}$ instead of $A$

$$
\begin{array}{ll}
\bar{A}_{12}+\bar{A}_{21} \geq \bar{A}_{33}, \quad & \bar{A}_{23} \geq \bar{A}_{21}+\bar{A}_{13}, \\
\bar{A}_{12}+\bar{A}_{21} \geq \bar{A}_{11}, & \bar{A}_{22} \leq \bar{A}_{12}+\bar{A}_{21},
\end{array}
$$

The face lattice of $\Psi(\mathcal{G})$ indexed by complete connected relations is displayed as solid graphs in Fig. 7. Blue arrows indicate subset inclusion. Red edges are those which belong to a cycle in the sink of the corresponding part. The six full-dimensional cones of $\mathcal{F}_{3}$ adjacent to $\Psi(\mathcal{G})$ are shown in lighter print next to $\mathcal{G}$. 


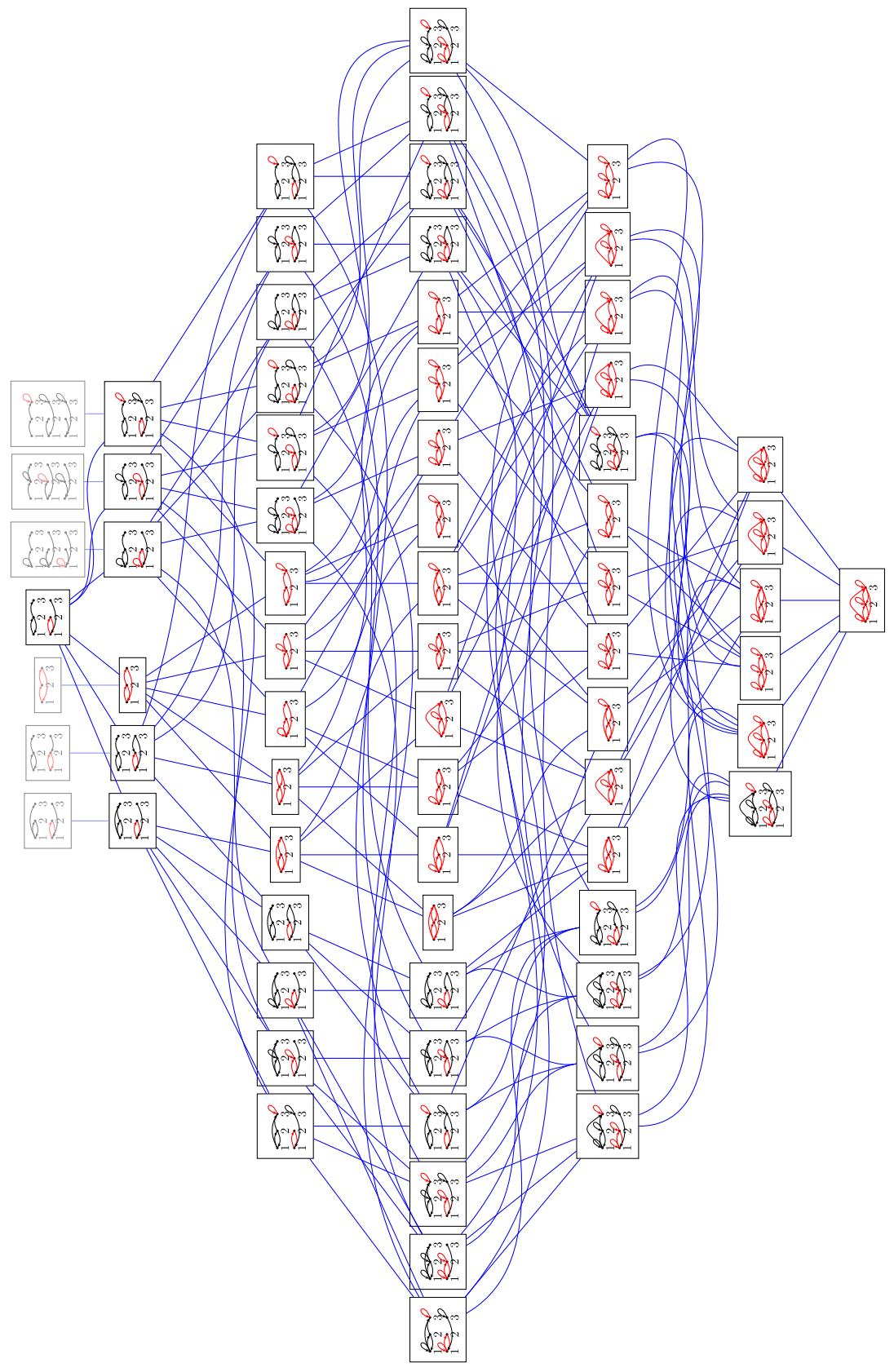

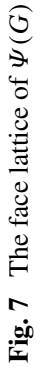


Acknowledgements The author would like to thank Bernd Sturmfels for helpful discussions. This research was supported by the DARPA Deep Learning program (FA8650-10-C-7020).

\section{References}

1. Ahuja, R.K., Magnanti, T.L., Orlin, J.B.: Network Flows: Theory, Algorithms, and Applications. Prentice Hall, New York (1993)

2. Baccelli, F., Cohen, G., Olsder, G.J., Quadrat, J.-P.: Synchronization and Linearity: An Algebra for Discrete Event Systems. Wiley, New York (1992)

3. Butkovič, P.: Max-linear Systems: Theory and Algorithms. Springer, Berlin (2010)

4. Cuninghame-Green, R.A.: Describing industrial processes with interference and approximating their steady-state behaviour. J. Oper. Res. Soc. 13(1), 95-100 (1962)

5. Develin, M., Sturmfels, B.: Tropical convexity. Doc. Math. 9, 1-27 (2004)

6. Elsner, L., van den Driessche, P.: Max-algebra and pairwise comparison matrices, ii. Linear Algebra Appl. 432(4), 927-935 (2010)

7. Hollings, C., Kambites, M.: Tropical matrix duality and Green's D relation. J. Lond. Math. Soc. 86(2), 520-538 (2012)

8. Joswig, M., Kulas, K.: Tropical and ordinary convexity combined. Adv. Geom. 10(2), 333-352 (2010)

9. Maclagan, D., Sturmfels, B.: Introduction to tropical geometry. Manuscript (2013)

10. Multiorder, S.S.: Kleene stars and cyclic projectors in the geometry of max cones. In: Contemporary Mathematics, vol. 495, pp. 317-342. Am. Math. Soc., Providence (2009)

11. Sergeev, S., Schneider, H., Butkovic, P.: On visualization scaling, subeigenvectors and Kleene stars in max algebra. Linear Algebra Appl. 431(12), 2395-2406 (2009)

12. Simon, I.: On semigroups of matrices over the tropical semiring. RAIRO Theor. Inform. Appl. 28(34), 277-294 (1994)

13. Sturmfels, B., Tran, N.M.: Combinatorial types of tropical eigenvectors. Bull. Lond. Math. Soc. 54, 27-36 (2013)

14. Tran, N.M.: Pairwise ranking: choice of method can produce arbitrarily different rank order. Linear Algebra Appl. 438, 1012-1024 (2013) 